PROCEEDINGS OF THE

AMERICAN MATHEMATICAL SOCIETY

Volume 128, Number 9, Pages 2687-2691

S 0002-9939(00)05338-7

Article electronically published on April 27, 2000

\title{
OPERATORS WITH BOUNDED CONJUGATION ORBITS
}

\author{
D. DRISSI AND M. MBEKHTA
}

(Communicated by David R. Larson)

\begin{abstract}
For a bounded invertible operator $A$ on a complex Banach space $X$, let $B_{A}$ be the set of operators $T$ in $\mathcal{L}(X)$ for which $\sup _{n \geq 0}\left\|A^{n} T A^{-n}\right\|<\infty$. Suppose that $S p(A)=\{1\}$ and $T$ is in $B_{A} \cap B_{A^{-1}}$. A bound is given on $\left\|A T A^{-1}-T\right\|$ in terms of the spectral radius of the commutator. Replacing the condition $T$ in $B_{A^{-1}}$ by the weaker condition $\left\|A^{-n} T A^{n}\right\|=o\left(e^{\epsilon \sqrt{n}}\right)$, as $n \rightarrow \infty$ for every $\epsilon>0$, an extension of the Deddens-Stampfli-Williams results on the commutant of $A$ is given.
\end{abstract}

\section{INTRODUCTION}

Let $\mathcal{L}(X), \mathcal{L}(H), S p(T)$, and $r(T)$ denote respectively the algebra of all bounded linear operators on a complex Banach space $X$, the algebra of all bounded linear operators on the complex separable infinite dimensional Hilbert space $H$, the spectrum of $T$, and the spectral radius of $T$. Let $A$ be an invertible operator in $\mathcal{L}(H)$. In 2 J. A. Deddens introduced the set

$$
B_{A}:=\left\{T \in \mathcal{L}(H): \sup _{n \geq 0}\left\|A^{n} T A^{-n}\right\|<\infty\right\} .
$$

It is easy to see that $B_{A}$ is an algebra which contains the commutant $\{A\}^{\prime}$ of $A$. In the case of finite dimensional Hilbert spaces, J. A. Deddens [2] showed that $B_{A}=$ $\{A\}^{\prime}$ if and only if there exists a nonzero scalar $\alpha$, such that $A=\alpha(I+N)$, with $N$ nilpotent. In the same paper Deddens conjectured that in the infinite dimensional case we have equality if the spectrum of $A$ is reduced to $\{1\}$. In 1980, J. P. Williams [10] proved that if the spectrum of $A$ is reduced to $\{1\}$, then $B_{A} \cap B_{A^{-1}}=\{A\}^{\prime}$.

In 1983, P. G. Roth [6] gave a negative answer to Deddens' conjecture. He showed the existence of a quasinilpotent operator $Q$ (the classical quasinilpotent Volterra integration operator) for which $B_{A} \neq\{A\}^{\prime}$ when $A=I+Q$.

In this paper considering a more general situation of a Banach space and following a different approach, we first intend to give a quantitative result (Theorem 4). As a corollary we obtain Williams' result. Subsequently, we improve Williams' result by replacing his condition on $A^{-1}$ by the weaker condition $\left\|A^{-n} T A^{n}\right\|=o\left(e^{\epsilon \sqrt{n}}\right)$, as $n \rightarrow \infty$ for every $\epsilon>0$. This could be the best possible result.

Received by the editors June 23, 1998 and, in revised form, October 27, 1998.

1991 Mathematics Subject Classification. Primary 47B10, 47B15.

Key words and phrases. Bounded conjugation orbit, spectrum, spectral radius.

The first author acknowledges support from Kuwait University.

(C)2000 American Mathematical Society 


\section{RESUlts}

Let $f$ be an entire function and let $M_{f}(r)=\max _{|z|=r}|f(z)|$. We say that $f$ is of finite order if there exists $k \geq 0$ such that

$$
M_{f}(r) \leq e^{r^{k}} \text { for } r \text { large. }
$$

The infimum of all $k$ satisfying this inequality is called the order of $f$ and denoted by $\tau(f)$. It is easy to verify that

$$
\tau(f)=\lim _{r \rightarrow \infty} \frac{\log \log M_{f}(r)}{\log r} .
$$

Now suppose that $f$ is an entire function of finite order $\tau(f)$. We define the type of $f$, denoted by $\sigma(f)$, to be the infimum of all nonnegative numbers $a$ such that

$$
M_{f}(r) \leq e^{a r^{\tau(f)}} .
$$

We then have

$$
\sigma(f)=\limsup _{r \rightarrow \infty} \frac{\log M_{f}(r)}{r^{\tau(f)}} .
$$

When $\sigma(f)=0$, we say that $f$ is of minimal type. If the entire function $f$ is of order at most one, then by [3 p. 84] (see also 4]), the type of $f$ is given by

$$
\sigma(f)=\limsup _{n \rightarrow \infty}\left|f^{(n)}(0)\right|^{\frac{1}{n}} .
$$

Lemma 1. Let $A \in \mathcal{L}(X)$. For each $u \in \mathcal{L}(X)^{*}$ and each $T$ in $\mathcal{L}(X)$, the entire function $\Phi: z \rightarrow u\left(e^{z A} T e^{-z A}\right)$ is of exponential type $r\left(\Delta_{A}(T)\right)$, where $\Delta_{A}(T)=$ $A T-T A$.

Proof. For $z \in \mathbb{C}$ and $T, A$ in $\mathcal{L}(X)$,

$$
\left|u\left(e^{z A} T e^{-z A}\right)\right| \leq\|u\| e^{(|z|\|A\|)}\|T\| e^{(|z|\|A\|)} .
$$

So, $M_{f}(r) \leq\|u\| e^{2 r\|A\|}\|T\|$, which gives us that the order of $u\left(e^{z A} T e^{-z A}\right)$ is less than or equal to 1 . The $n$-th derivative of $\Phi(z)$ at zero is $u\left(\Delta_{A}^{n}(T)\right)$. Thus by Levin's theorem (see [3], p. 84) or equation 2.2.12 in Boas (1], p.11) the type of $\Phi(z)$ is equal to $\lim _{\sup _{n \rightarrow \infty}}\left|u\left(\Delta_{A}^{n}(T)\right)\right|^{\frac{1}{n}}$, which is less than or equal to the spectral radius of $\Delta_{A}(T)$.

The next lemma is a fundamental tool needed in the proof of one of the main results in this paper. Its proof given below is included mainly in order to keep this paper as self-contained as possible. The result is a consequence of the well-known theorem of Bernstein, that is, an entire function of minimal type is not bounded on the real line unless it is a constant.

Lemma 2. An entire function $f$ of growth $\left(\frac{1}{2}, 0\right)$ is not bounded on any half-line unless it is a constant.

Proof. Take $g(z)=f\left(z^{2}\right)$. Then $g$ is an entire function of growth $(1,0)$ and is bounded on the real line. So, by Bernstein's theorem $g$ must be constant.

Lemma 3. Let $f$ be an entire function of minimal type. Suppose that

(i) $|f(t)| \leq M$, for all $t \geq 0$, and

(ii) $|f(-t)|=o\left(e^{\epsilon \sqrt{t}}\right)$ as $t \rightarrow \infty$ for every $\epsilon>0$.

Then $f$ is a constant function. 
Proof. Let $\Pi_{+}:=\{z \in \mathbb{C}: \operatorname{Re}(z)>0\}$ and $\Pi_{-}:=\{z \in \mathbb{C}: \operatorname{Re}(z)<0\}$. For $\epsilon>0$ and $z \in \Pi_{+}$let $g_{\epsilon}(z)=e^{-\epsilon \sqrt{z}} f(i z)$. Thus $g_{\epsilon}$ is an analytic function on $\Pi_{+}$and continuous on the closure of $\Pi_{+}$, such that

$$
\left|g_{\epsilon}(z)\right|=e^{-\epsilon \operatorname{Re}(\sqrt{z})}|f(i z)|=e^{-\epsilon|z|^{\frac{1}{2}} \cos \left(\frac{1}{2} \operatorname{Arg}(z)\right)}|f(i z)|,
$$

where $\operatorname{Arg}(z)$ is the determination of the argument of $z$ in $(-\pi, \pi)$. Since $\cos \left(\frac{1}{2} \operatorname{Arg}(z)\right) \geq 0$ for $z \in \Pi_{+}$, we have

$$
\left|g_{\epsilon}(z)\right| \leq|f(i z)|, \quad \text { for } z \in \Pi_{+} .
$$

On the other hand, since $f$ is of minimal type, we have for an arbitrary $\epsilon>0$,

$$
\left|g_{\epsilon}(z)\right| \leq C e^{a|z|} \text {, as }|z| \rightarrow \infty, z \in \Pi_{+} .
$$

Moreover, for $z$ on the imaginary axis, we have

$$
\left|g_{\epsilon}(i t)\right|=e^{-\epsilon\left(\sqrt{\frac{|t|}{2}}\right)}|f(-t)| .
$$

Condition (ii) implies the existence of $K_{\epsilon}>0$ for which

$$
|f(t)| \leq K_{\epsilon} e^{\epsilon\left(\sqrt{\frac{|t|}{2}}\right)}, \quad \text { for every real } t \text { and every } \epsilon>0 .
$$

Hence $g_{\epsilon}$ is bounded on the imaginary axis. It follows by a standard PhragménLindelöf argument that $g_{\epsilon}$ is bounded on the closure of $\Pi_{+}$(see [7], p. 282). Thus $|f(i z)|=\left|e^{\epsilon \sqrt{z}} g_{\epsilon}\right| \leq K_{\epsilon} e^{\epsilon \sqrt{z}}$. So,

$$
\limsup _{|z| \rightarrow \infty, z \in \Pi_{+}} \frac{\log |f(i z)|}{|i z|^{\frac{1}{2}}}=0 .
$$

Similarily, we obtain for $f(-z)$

$$
\limsup _{|z| \rightarrow \infty, z \in \Pi_{-}} \frac{\log |f(i z)|}{|i z|^{\frac{1}{2}}}=0 .
$$

Consequently, $f$ is an entire function of growth $\left(\frac{1}{2}, 0\right)$. By Lemma 2 we obtain that $f$ is constant.

Our main results are the following.

Theorem 4. Let $S$ be in $\mathcal{L}(X)$ and suppose that $T$ is in $B_{e^{S}} \cap B_{e^{-S}}$. Then

$$
\left\|e^{S} T e^{-S}-T\right\| \leq 2 \tan \left(\frac{r\left(\Delta_{S}\right)}{2}\right) C \quad \text { if } r\left(\Delta_{S}\right) \leq 2 \pi,
$$

where $C=\sup _{n \geq 0}\left\|e^{n S} T e^{-n S}\right\|<\infty$.

Proof. Let $f(z)=u\left(e^{z S} T e^{-z S}\right)$, where $u$ is a functional of norm one on $\mathcal{L}(X)$. Condition $T \in B_{e^{S}} \cap B_{e^{-S}}$ implies that $f$ is bounded on the real axis. On the other hand, $f(z)=u\left(\sum_{n=0}^{\infty} \frac{z^{n}}{n !} \Delta_{S}^{n}(T)\right)$. So, By Lemma $1, f$ is an entire function of exponential type $r\left(\Delta_{S}(T)\right)$. Hence, if $r\left(\Delta_{S}(T)\right)<\pi$, then by Bernstein's theorem [1, Theorem 11.4.1, p. 214], we obtain

$$
\left|u\left(e^{S} T e^{-S}-T\right)\right|=|f(1)-f(0)| \leq 2 \sup _{t \in \mathbb{R}}|f(t)| \tan \left(\frac{r\left(\Delta_{S}(T)\right)}{2}\right) .
$$

By applying Hahn-Banach's theorem we obtain the desired result. 
As a consequence we obtain the following result of Deddens-Stampfli-Williams.

Corollary 5. If $Q$ is a quasinilpotent operator in $\mathcal{L}(X)$, then $B_{(I+Q)} \cap B_{(I+Q)^{-1}}=$ $\{I+Q\}^{\prime}$.

We also have the following improvement of Williams's result which we claim as the best possible result. The proof was inspired to us by the articles [5] and 9 .

Theorem 6. Let $Q$ be a quasinilpotent operator in $\mathcal{L}(X)$. Suppose that

$$
\left\|e^{-n(I+Q)} T e^{n(I+Q)}\right\|=o\left(e^{\epsilon \sqrt{n}}\right), \quad \text { as } n \rightarrow \infty \text { for every } \epsilon>0 .
$$

Then $T \in B_{I+Q}$ implies $T \in\{I+Q\}^{\prime}$.

Proof. Apply Lemma 3 to the function $f(z)=u\left(e^{z(I+Q)} T e^{-z(I+Q)}\right)$, where $u$ is a functional of norm 1 .

Theorem 7. Let $A$ be an invertible operator in $\mathcal{L}(X)$. Suppose that

$$
C=\sup _{n \geq 0}\left\|e^{n A} T e^{-n A}\right\|<\infty .
$$

Then

$$
\|A T-T A\| \leq \frac{2 C}{e} \limsup _{n \rightarrow \infty} n\left(\left\|\Delta_{A}^{n}(T)\right\|\right)^{\frac{1}{n}} .
$$

Proof. Let us consider the function $f(z)=u\left(e^{z^{2} A} T e^{-z^{2} A}\right)$, where $u$ is a functional of norm one. Then $f$ is an entire function of order less than or equal to one. So, by Levin's theorem [3, p. 84] $f$ is of exponential type

$$
\sigma(f)=\limsup _{n \rightarrow \infty}\left|f^{(n)}(0)\right|^{\frac{1}{n}} \leq \limsup _{k \rightarrow \infty}\left(\frac{(2 k) !}{k !}\right)^{\frac{1}{2 k}}\left\|\Delta_{A}^{k}(T)\right\|^{\frac{1}{2 k}} .
$$

Applying Stirling's formula, we obtain $\sigma(f) \leq \frac{2}{\sqrt{e}} \sqrt{\limsup _{n \rightarrow \infty} n\left(\left\|\Delta_{A}^{n}(T)\right\|\right)^{\frac{1}{n}}}$.

On the other hand, the hypothesis implies the boundedness of $f$ on the real axis. Hence, by Bernstein's inequality for entire functions, we obtain

$$
\left|f^{\prime \prime}(0)\right|=2|u(A T-T A)| \leq \sup _{t \in \mathbb{R}}(\sigma(f))^{2}=\frac{4 C}{e} \limsup _{n \rightarrow \infty} n\left(\left\|\Delta_{A}^{n}(T)\right\|\right)^{\frac{1}{n}} .
$$

Remark. As the reader may have noticed, all these results are valid in the general situation of Banach algebras.

\section{REFERENCES}

[1] R. P. Boas : Entire Functions, Academic Press, New York, 1954. MR 16:914f

[2] J. A. Deddens : Another description of nest algebras in Hilbert spaces operators, Lecture notes in Mathematics No. 693, (pp. 77-86), Springler-Verlag, Berlin, 1978. MR 80f:47033

[3] B. Ja. Levin : Distributions of Zeros of Entire Functions, Amer. Math. Soc. Providence, 1964. MR 28:217

[4] B. Ja. Levin : Lectures on Entire Functions, Translations of Mathematical Monographs, Vol. 150, American Mathematical Society, 1996. MR 97j:30001

[5] G. Lumer and R. S. Phillips : Dissipative operators in a Banach space, Pacific J. Math. 11(1961), 679-698. MR 24:A2248

[6] P. G. Roth : Bounded orbits of conjugation, analytic theory, Indiana Univ. Math. J., 32 (1983), 491-509. MR 85c:47039

[7] W. Rudin : Real \& Complex Analysis, Mc Graw-Hill, New York, 1966. MR 35:1420 
[8] J. G. Stampfli : On a question of Deddens in Hilbert space operators, Lecture Notes in Mathematics No. 693, (pp. 169-173), Springer-Verlag, Berlin, 1978. MR 80f:47034

[9] J. G. Stampfli and J. P. Williams : Growth conditions and the numerical range in a Banach algebra, Tôhôku Math. J. 20(1968), 417-424. MR 39:4674

[10] J. P. Williams : On a boundedness condition for operators with singleton spectrum, Proc. Amer. Math. Soc., 78(1980), 30-32. MR 81k:47008.

Department of Mathematics and Computer Science, Faculty of Science, Kuwait University, P. O. Box 5969, Safat 13060, Kuwait

E-mail address: drissi@math-1.sci.kuniv.edu.kw

URA 751 au CNRS \& UFR de Mathematiques, Université de Lille I, F-59655, VilLENEUVE D'ASQ, France

Université de Galatasaray, Ciragan Cad no. 102, Ortakoy 80840, Istanbul, Turquie

E-mail address: Mostafa.Mbekhta@univ-lille1.fr 